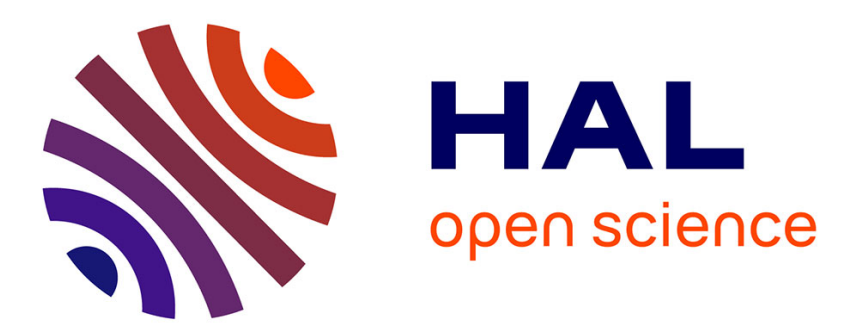

\title{
MÉTHODE COMPOSITE POUR LE CALCUL DE LA RÉPONSE IMPULSIONNELLE D'UNE SPHÈRE RIGIDE
}

\author{
Z. Sun, G. Gimenez, O. Basset, F. Denis
}

\section{- To cite this version:}

Z. Sun, G. Gimenez, O. Basset, F. Denis. MÉTHODE COMPOSITE POUR LE CALCUL DE LA RÉPONSE IMPULSIONNELLE D'UNE SPHÈRE RIGIDE. Journal de Physique Colloques, 1990, 51 (C3), pp.C3-231-C3-240. 10.1051/jphyscol:1990324 。 jpa-00230752

HAL Id: jpa-00230752

https://hal.science/jpa-00230752

Submitted on 1 Jan 1990

HAL is a multi-disciplinary open access archive for the deposit and dissemination of scientific research documents, whether they are published or not. The documents may come from teaching and research institutions in France or abroad, or from public or private research centers.
L'archive ouverte pluridisciplinaire HAL, est destinée au dépôt et à la diffusion de documents scientifiques de niveau recherche, publiés ou non, émanant des établissements d'enseignement et de recherche français ou étrangers, des laboratoires publics ou privés. 
COLLOQUE DE PHYSIQUE

Colloque C3, supplément au $\mathrm{n}^{\circ} 17$, Tome 51, ler septembre 1990

\section{MÉTHODE COMPOSITE POUR LE CALCUL DE LA RÉPONSE IMPULSIONNELLE D'UNE SPHÈRE RIGIDE}

Z. SUN, G. GIMENEZ, O. BASSET et F. DENIS

Laboratoire "Traitement du signal et Ultrasons" CNRS URA-1216, INSA

Bât. 502, F-69621 Villeurbanne Cedex, France

Résumé - Dans ce travail nous proposons une méthode pratique pour calculer la réponse acoustique d'une sphère rigide. Cette méthode utilise conjointement la méthode de l'optique physique, qui donne de bons résultats en haute fréquence, et la méthode modale, qui convient au calcul en basse fréquence. Cette combinaison nous permet de nous affranchir des problèmes liés à l'utilisation d'une seule de ces méthodes : inadéquation de la méthode de l'optique physique en basse fréquence et difficultés de convergence de la méthode modale en haute fréquence. Actuellement nous calculons la réponse impulsionnelle (réponse à un Dirac) d'une sphère rigide dans une situation de rétrodiffusion.

Abstract - In this paper we propose a practical method to calculate the acoustical response of rigid sphere. This method combines the physical optic method, which gives good results at high frequences, with the modal method, which is suitable at low frequencies. This gets around the problems that arise when only one of these methods is used, namely the failure of the physical optic method at low frequencies and the convergence difficulties of the modal method at high frequencies. Here, we calculate the impulse response (response to a Dirac pressure transmission) of a rigid sphere for a backscattering situation.

\section{I - Introduction}

La diffusion acoustique par une sphère rigide est un problème classique. Beaucoup de travaux $y$ ont été consacrés[1] du fait de sa simplicité relative, de ses intérêts physique et mathématique, et de ses nombreuses applications. Pour traiter le problème de la diffusion en réponse à une impulsion, deux voies peuvent être empruntées : travailler directement dans le domaine temporel ou travailler d'abord dans le domaine fréquentiel puis retourner dans le domaine temporel par transformée de Fourier inverse. Peu de travaux existe sur la première possibilité. Par contre, dans le domaine fréquentiel, diverses méthodes ont été étudiées. Cependant, travailler en haute fréquence est souvent pénible et fastidieux. Bien que différentes techniques puissent être employées pour surmonter les difficultés dues à la convergence et à l'instabilité numérique, la réponse impulsionnelle est toujours difficile à déduire de la fonction de transfert puisque cela nécessite la connaissance de cette dernière dans une plage de fréquences s'étendant de zéro à l'infini. Cette condition n'est remplie dans aucun cas pratique.

Dans ce travail nous intéressons à la rétrodiffusion d'une sphère rigide recevant une impulsion de pression en forme de Dirac émise par une source ponctuelle. Sachant que la méthode analytique usuelle, avec laquelle le champ diffusé par une sphére rigide est exprimé en séries de Rayleigh, converge rapidement en basse fréquence tandis que la méthode de l'optique physique donne satisfaction en haute fréquence, nous allons introduire, en étudiant la différence fréquentielle entre les résultats donnés par ces deux méthodes, une approche approximative pour calculer la réponse impulsionnelle. nous donnerons alors la réponse temporelle à quelques autres signaux en les convoluant avec la réponse impulsionnelle obtenue. 


\section{II - Champ diffusé calculé par la méthode de l'optique physique}

Nous considérons une onde de pression incidente sphérique émise par une source ponctuelle $S$ :

$$
P_{i}=\frac{1}{4 \pi r} e^{i(k r-\omega t)}
$$

où $\mathrm{k}=\omega / \mathrm{c}=2 \pi / \mathrm{c}$ est le nombre d'onde, $\mathrm{c}$ la célérité acoustique dans le milieu fluide, $\mathrm{r}$ la distance

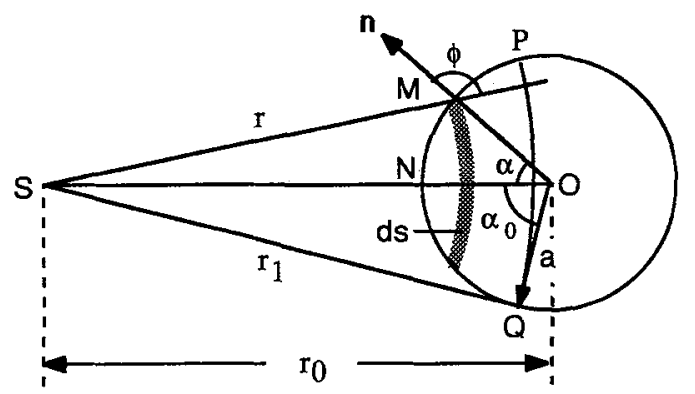

Figure 1: Géométrie de diffusion et indication des paramètres.

entre $S$ et le point courant du champ, tandis que $f$ et $\omega$ sont respectivement la fréquence et la fréquence angulaire. Il est bien connu que l'expression (1) est la solution de l'équation d'Helmholtz suivante (dans l'espace libre) :

$$
\left(\nabla^{2}+k^{2}\right) P_{i}=-e^{i \omega t} \delta(r)
$$

qui correspond à une émission en $\mathrm{e}^{-\mathrm{i} \omega \mathrm{t}}$ de la source $\mathrm{S}$, où $\nabla^{2}$ est l'opérateur de Laplace. Cette onde de pression est diffusée par une sphère rigide de rayon a dont le centre est situé au point $O$, comme l'illustre la figure1. Soit $\mathrm{r}_{0}$ la distance de $\mathrm{S}$ à $\mathrm{O}$. Si on ne s'intéresse qu'au cas de rétrodiffusion, c'est à dire quand le récepteur est au même point que la source $S$, le champ diffusé au point récepteur est alors donné par l'équation d'Helmholtz-Kirchhoff ${ }^{[2]}$ :

$$
P_{S}(f)=\frac{1}{4 \pi} \int_{S}\left\{P \frac{\partial}{\partial n}\left(\frac{e^{i k r}}{r}\right)-\frac{e^{i k r}}{r} \frac{\partial P}{\partial n}\right\} d S
$$

où $P$ est la pression totale en un point quelconque $M$ de la surface $S$ de la sphère tandis que $r$ est la distance du point $M$ au récepteur (S). L'opération $\partial / \partial \mathrm{n}$ représente la dérivation suivant la direction du vecteur unitaire $\mathbf{n}$, normal à la surface et orienté vers l'extérieur. L'intégration est à réaliser sur la surface totale de la sphère.

Pour une sphère rigide, la condition à limite de type Neumman doit être adoptée :

$$
\frac{\partial P}{\partial n}=0 \quad \text { sur la surface }
$$

L'approximation de la méthode de l'optique physique suppose que, pour un objet rigide, la pression acoustique à la surface est [3]: 


$$
P=\left\{\begin{array}{lc}
2 P_{i} & \text { sur la partie éclairée de la surface } \\
0 & \text { sur la partie de la surface dans l'ombre }
\end{array}\right.
$$

D'où, en utilisant (3) et (4) nous avons

$$
P_{0}(f)=\frac{1}{16 \pi^{2}} \int_{\Sigma} \frac{e^{i k r}}{r} \frac{\partial}{\partial n}\left(\frac{e^{i k r}}{r}\right) d S
$$

Ici nous avons remplacé $P_{\mathrm{s}}(\mathrm{f})$ par $\mathrm{P}_{\mathrm{o}}(\mathrm{f})$ afin de spécifier que ce résultat est obtenu par la méthode de l'optique physique. L'intégrale (6) est calculée sur la surface éclairée $\Sigma$ qui est délimitée par un cercle correspondant à l'angle [cf. Fig. (1)] :

$$
\alpha_{0}=\arccos \left(\mathrm{a} / \mathrm{r}_{0}\right)
$$

et (6) peut alors s'exprimer par :

$$
P_{0}(f)=\frac{a}{8 \pi r_{0}\left(r_{0}-a\right)} e^{i 2 k(r 0-a)}-\frac{1}{8 \pi r_{0}} \int_{r_{0}-a}^{r_{1}} \frac{e^{i} 2 k r}{r} d r
$$

où

$$
\mathrm{r}_{1}=\left(\mathrm{r}_{0}^{2}-\mathrm{a}^{2}\right)^{1 / 2}
$$

est la longueur de la tangente de $S$ à la sphère.

Nous savons que l'intégrale :

$$
\int_{-\infty}^{+\infty} P_{i}(f) d f=\int_{-\infty}^{+\infty} \frac{1}{4 \pi r} e^{i(k r-\omega t)} d f=\frac{1}{4 \pi r} \delta(t-r / c)
$$

est la solution de l'équation de D'Alembert suivante [4] :

$$
\left(\nabla^{2}-c^{-2} \frac{\partial^{2}}{\partial t^{2}}\right) p(r, t)=-\delta(t) \delta(r)
$$

Elle décrit une impulsion de pression en forme de Dirac émise du point $\mathrm{S}$. Le système considéré etant linéaire, la réponse impulsionnelle d'une sphère rigide peut être obtenue en intégrant l'équation (8):

$$
\begin{aligned}
& p_{0}(t)=\int_{-\infty}^{+\infty} P_{0}(f) e^{-i \omega t} d f \\
& =\frac{a}{8 \pi r_{0}\left(r_{0}-a\right)} \delta\left(t-\tau_{1}\right)-\frac{1}{8 \pi r_{0} t} \operatorname{rect}\left(\frac{t-\left(\tau_{1}+\tau_{2}\right) / 2}{\tau_{2}-\tau_{1}}\right)
\end{aligned}
$$

où

$$
\tau_{1}=\frac{2\left(\mathrm{r}_{0}-\mathrm{a}\right)}{\mathrm{c}}, \quad \tau_{2}=\frac{2 \mathrm{r}_{1}}{\mathrm{c}}
$$


et rect représente la fonction rectangle de largeur $\left(\tau_{2}-\tau_{1}\right)$, centrée au point $t=\left(\tau_{1}+\tau_{2}\right) / 2$. Il est évident que $\tau_{1}$ est la durée du trajet aller-retour entre $S$ et le pôle $N$, tandis que $\tau_{2}$ est celle du trajet entre $S$ et $Q$.

La figure 2 montre la réponse donnée par la relation (12), calculées pour $\mathrm{a}=0,5 \mathrm{~cm}, \mathrm{r}_{0}=20 \mathrm{~cm}$ et $c=331 \mathrm{~m} / \mathrm{s}$. Lorsque $\mathrm{r}_{0}$ est très grand devant $\mathrm{a}$, la réponse fréquentielle (8) se réduit à :

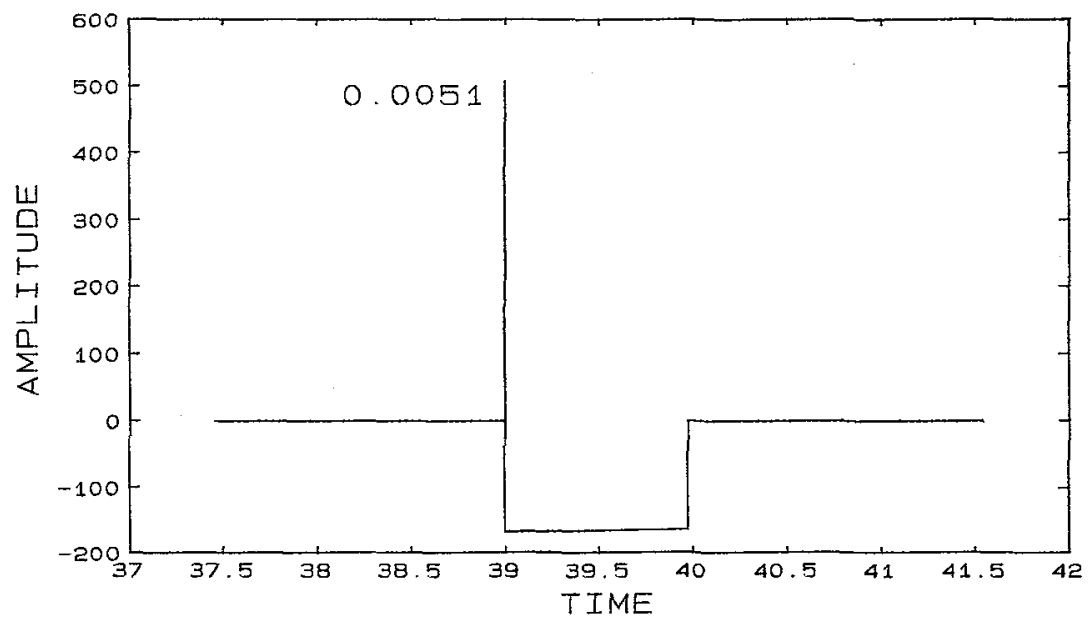

Figure 2: Réponse impulsionnelle (pression rétrodiffusée) pour une sphère rigide $(\mathrm{a}=0,5 \mathrm{~cm}$, $\mathrm{r}_{0}=20 \mathrm{~cm}, \mathrm{c}=331 \mathrm{~m} / \mathrm{s}$ ) obtenue par la méthode de l'optique physique.

$$
\left.P_{0}(f)=\frac{a}{8 \pi r_{0}\left(r_{0}-a\right)}\left[e^{i \omega \tau}\right)-\frac{\sin \frac{\omega a}{c}}{\frac{\omega a}{c}} e^{i \omega\left(\tau_{1}+\tau_{2}\right) / 2}\right]
$$

En conséquence, dans le domaine temporel et compte tenu, du fait que $\tau_{2}-\tau_{1} \ll \tau_{1}$, l'expression (12) peut alors être reécrite :

$$
p_{0}(t)=\frac{a}{8 \pi r_{0}\left(r_{0}-a\right)} \delta\left(t-\tau_{1}\right)-\frac{c}{16 \pi r_{0}\left(r_{0}-a\right)} \operatorname{rect}\left(\frac{t-\left(\tau_{1}+\tau_{2}\right) / 2}{\tau_{2}-\tau_{1}}\right) .
$$

Il est aisé de vérifier que (14) et (15) représentent une paire de transformées de Fourier.

\section{III - Méthode modale}

Maintenant nous examinons le système décrit par la Fig.(1) en coordonnées sphériques [Fig.(3)]. En considérant qu'il y a symétrie autour de l'axe $z$, l'onde de pression sphérique incidente en un point $M$ est donnée par [5]

$$
P i=\frac{e^{i k R}}{4 \pi R}=\frac{i k}{4 \pi} \sum_{n=0}^{\infty}(2 n+1)(-1)^{n} h_{n}^{(1)}\left(k r_{0}\right) j_{n}(k r) P_{n}(\cos \theta),
$$

et l'onde diffusée peut alors être exprimée ${ }^{[6]}$ : 


$$
P_{S}(f)=\frac{i}{4 \pi} \sum_{n=0}^{\infty} A_{n}(2 n+1)(-1)^{n} h_{n}^{(1)}(k r) P_{n}(\cos \theta)
$$

où les $h_{n}(1)$ sont les fonctions d'Hankel sphériques de première espèce, d'ordre $n$. Elles sont choisies pour obtenir des ondes diffusées divergentes. Les fonctions $j_{n}$ et $P_{n}$ sont respectivement

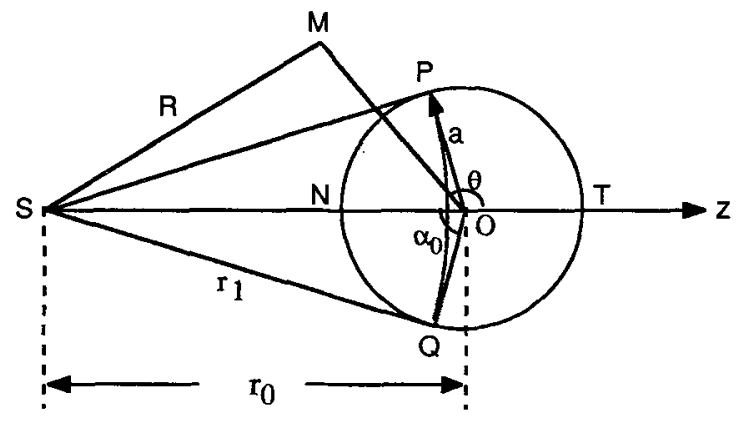

Figure 3: La sphère rigide de rayon a est centrée sur l'origine $O$ des coordonnées. L'onde sphérique est transmise par une source ponctuelle $S$ située sur la partie négative de l'axe $\mathrm{z}$ à une distance $\mathrm{r}_{0}$ de l'origine.

les fonctions de Bessel sphériques et les fonctions de Legendre de première espèce, d'odre $\mathrm{n}$, tandis que An est un coefficient à déterminer à partir des conditions aux limites.

Le champ total est :

$$
\mathbf{P}=\mathbf{P}_{\mathrm{i}}+\mathbf{P}_{\mathbf{S}}
$$

Pour une sphère entourée d'un fluide non visqueux, la condition sur la surface s'écrit :

$$
\left.\mathrm{u}_{\mathrm{r}}\right|_{\mathrm{r}=\mathrm{a}}=0
$$

où $u_{\mathrm{r}}$ dénote le déplacement radial sur la surface.

Soient $\Phi$ le potentiel de vitesse, $\vee$ la vitesse de vibration des particules, $u$ le déplacement des particules et $\rho$ la masse volumique du matériau fluide. En considérant une onde incidente monochromatique, on a alors :

$$
\begin{aligned}
& P=\rho \frac{\partial \Phi}{\partial t}=(-i \omega) \rho \Phi \\
& v=\frac{\partial u}{\partial t}=(-i \omega) \mathbf{u} \\
& v=-\operatorname{grad} \Phi .
\end{aligned}
$$

Il en résulte :

$$
\mathbf{u}=\frac{1}{\rho \omega^{2}} \operatorname{grad} \mathrm{P},
$$

et, en tenant compte de la situation de rétrodiffusion $\left(r=r_{0}, \theta=\pi\right)$, nous déduisons de (16), (17), (18), (19) et (21) que : 


$$
A_{n}=-\frac{k h_{n}^{(1)}\left(k r_{0}\right) j_{n}^{\prime}(k a)}{4 \pi h_{n}^{(1)}(k a)}
$$

Le champ de la pression rétrodiffusée est alors donné par :

$$
P_{s}(f)=-\frac{i k}{4 \pi} \sum_{n=0}^{\infty}(2 n+1) \frac{\left[h_{n}^{(1)}\left(k r_{0}\right)\right]^{2} j^{\prime}(k a)}{4 \pi h_{n}^{(1)}(k a)}
$$

Cette expression décrit la réponse fréquentielle du système. Pour ce calcul, les termes jusqu'à $\mathrm{n}=60$ ont été pris en compte. Une analyse de convergence de (23) montre que pour $\mathrm{ka}<50$, cet ordre de 60 est suffisant pour que l'erreur relative (écart relatif entre la valeur approximée et la valeur exacte) soit inférieure à $10^{-6}$.

\section{IV - Réponse impulsionnelle d'une sphère rigide}

En exprimant le champ incident sous la forme (1) ou (16), on obtient la téponse impulsionnelle :

$$
p_{s}(t)=\int_{-\infty}^{+\infty} P_{s}(f) e^{-i 2 \pi f t} d f
$$

Pour des raisons pratiques, nous la mettons sous la forme usuelle :

$$
p_{s}(t)=\int_{-\infty}^{+\infty} P_{s 1}(f) e^{i 2 \pi f 1} d f
$$

avec :

$$
P_{s 1}(f)=P_{s}(-f)
$$

Comme $\mathrm{p}_{\mathrm{s}}(\mathrm{t})$ est un signal physique (nécessairement réel), sa transformée de Fourier $\mathrm{P}_{\mathrm{s} 1}(\mathrm{f})$, définie par :

$$
P_{s 1}(f)=\int_{-\infty}^{+\infty} p_{s}(t) \cdot e^{-i 2 \pi f t} d t
$$

vérifie

$$
P_{s 1}(-f)=P_{s 1}^{*}(f)
$$

où "*" dénote la conjugaison complexe de la fonction, et $\mathrm{P}_{\mathrm{s} 1}(\mathrm{f})$ peut être écrit comme :

$$
P_{s 1}(f)=P_{s 1}(|f|) U(f)+P_{s 1}^{*}(\mid f) U(-f)
$$

U(f) étant l'échelon unitaire. Par conséquent, prenant en considération (26), nous avons : 


$$
P_{s 1}(f)=P_{s}(|f|) U(-f)+P_{s}^{*}(|f|) U(f) \quad[\text { cf. Eq.(23)] }
$$

La même discussion peut être reprise pour $\mathrm{P}_{\mathrm{o} 1}(\mathrm{f})$. En définissant

$$
P_{01}(f)=P_{0}(-f)
$$

nous avons :

$$
p_{0}(t)=\int_{-\infty}^{+\infty} P_{01}(f) e^{i 2 \pi f t} d f
$$

avec

$$
P_{01}(f)=P_{0}(|f|) U(-f)+P_{0}^{*}(|f|) U(f)
$$

La figure 4 donne la partie réelle de la différence entre $\mathrm{P}_{\mathrm{s} 1}(\mathrm{f})$ et $\mathrm{P}_{\mathrm{o} 1}(\mathrm{f})$ pour ka variant de 0 à 46 et $\mathrm{a}=0,5 \mathrm{~cm}, \mathrm{r}_{0}=20 \mathrm{~cm}$ et $\mathrm{c}=331 \mathrm{~m} / \mathrm{s}$. Visiblement, quand la fréquence augmente, la différence entre $P_{s 1}(f)$ et $P_{01}(\dot{f})$ tend vers zéro. Ceci suggère que, pourvu que les fréquences soient suffisamment élevées, $P_{s 1}(f)$ peut être remplacée par $P_{o 1}(f)$. Autrement dit, en haute fréquence, nous pouvons nous servir du résultat de la méthode de l'optique physique au lieu de celui de la méthode modale. En supposant que la différence $P_{s_{1}}(f)-P_{o 1}(f)$ soit nulle pour ka supérieur à 46 :

$$
P_{s 1}(f)-P_{o 1}(f)=0 \quad \text { pour } \mathrm{ka} \geq 46
$$

la réponse impulsionnelle peut être maintenant calculée:

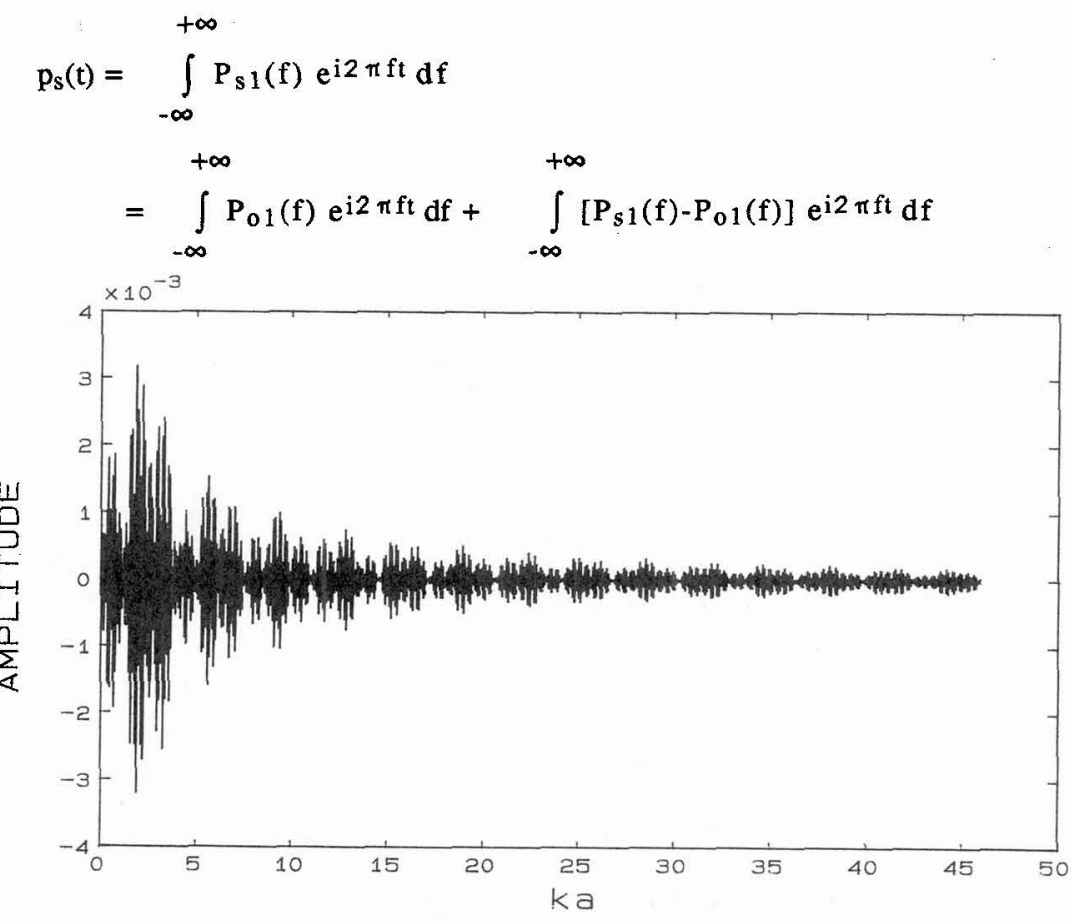

Figure 4: Partie réelle de la différence entre le résultat de la méthode modale et celui de la méthode de l'optique physique. 


$$
\approx p_{0}(t)+\int_{-f_{l}}^{+f_{l}}\left[P_{s 1}(f)-P_{01}(f)\right] e^{i 2 \pi f t} d f
$$

avec

$$
\mathrm{f}_{l}=46 \mathrm{c} / 2 \pi \mathrm{a}
$$

où $p_{0}(t)$ est donnée par (12), $P_{s 1}(f)$ par (23) et $(30), P_{01}(f)$ par (8) et (33). Notons que lorsque $r_{0} \ll a, p_{0}(t)$ et $P_{o}(f)$ peuvent être obtenues d'une façon plus simple à partir de (14) et (15). La figure 5 indique la réponse impulsionnelle (i.e. la réponse à un Dirac incident) pour $a=0,5 \mathrm{~cm}$, $r_{0}=20 \mathrm{~cm}$ et $\mathrm{c}=331 \mathrm{~m} / \mathrm{s}$. Afin de rendre plus claire la représentation, nous utilisons la durée $\tau=$ $2 \mathrm{a} / \mathrm{c}$ comme unité de temps dans toutes les figures qui décrivent des signaux temporels. Ici $\tau$ est le temps nécessaire à l'éclairement de la sphère par un Dirac incident. La réponse impulsionnelle commence par un Dirac à $\mathrm{t}=39 \tau$, dont l'amplitude relative, par rapport à celle de l'émission est $5,10 \times 10^{-3}$. Cette partie correspond à une réflexion de l'onde acoustique incidente au point brillant $\mathbf{N}$ sur la sphère [cf. Fig.(3)]. La seconde partie, i.e. celle qui commence par un pic négatif, est dominée principalement par la diffraction. La troisième partie de la réponse, qui se manifeste comme un pic positif, représente les ondes rampantes. Ces ondes rampantes sont engendrées sur le cercle correspondant à l'angle $\alpha_{0}$ [cf. Eq. (7)]. Comme nous pouvons le voir, la distance temporelle entre le Dirac et le maximum du pic positif est environ $\tau_{r}=2,636 \tau$, qui correspond à la différence entre les chemins $\overline{S N}+\overline{N S}$ et $\overline{S P}+P T Q+\overline{Q S}$. Dans le cas où $a=0,5 \mathrm{~cm}, \mathbf{r}_{0}=20 \mathrm{~cm}$ et $\mathrm{c}=331 \mathrm{~m} / \mathrm{s}$, nous avons :

$$
\left[\frac{\overline{S P}+P T Q+\overline{Q S}}{c}-\frac{\bar{S} \bar{N}+\bar{N} \bar{S}}{c}\right] \simeq 2,571 \tau
$$

qui est plus petit que $\tau_{r}$. Ceci signifie que la vitesse de phase des ondes rampantes qui se propagent le long de la partie dans l'ombre de la surface, est légèrement inférieure à celle des ondes acoustiques se propageant dans le milieu environnant. Dans cette figure on peut aussi remarquer des petites oscillations de faible amplitude vers le milieu de la courbe. Cela est dû à la perte d'information imposée par (34) pour les fréquences au-delà de $f= \pm f_{l}$. En lissant ces oscillations, il est possible d'aboutir à un résultat plus exact.

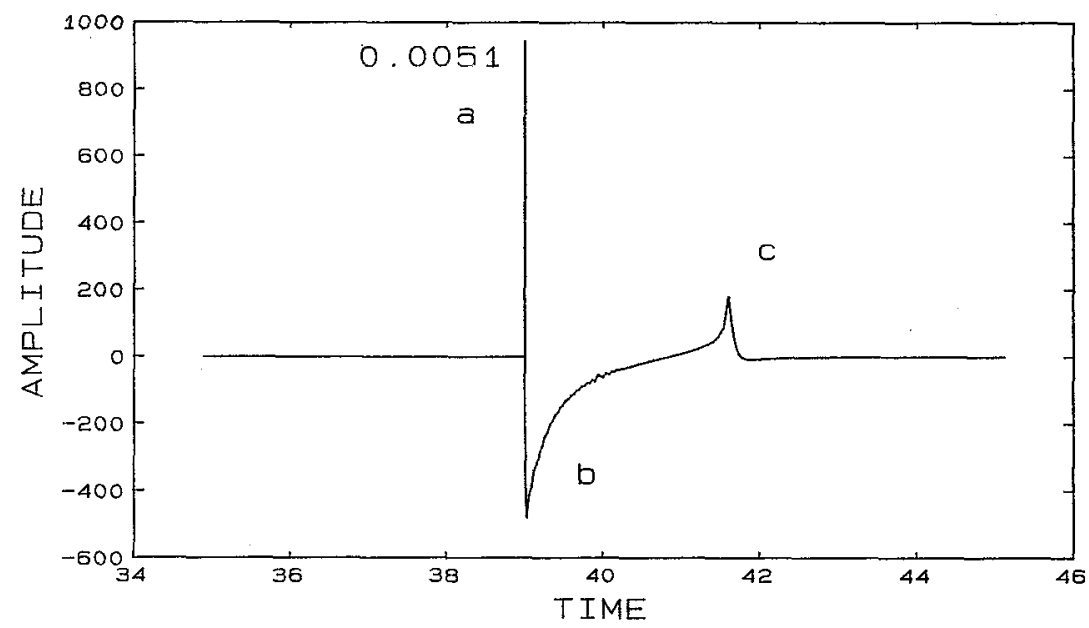

Figure 5: Réponse impulsionnelle d'une sphère rigide dans une situation de rétrodiffusion $\left(\mathrm{a}=0.5 \mathrm{~cm}, \mathrm{r}_{0}=20 \mathrm{~cm}, \mathrm{c}=331 \mathrm{~m} / \mathrm{s}\right.$ ) 


\section{Application au calcul des réponses à divers signaux}

Après avoir obtenu la réponse impulsionnelle $p_{s}(t)$, la réponse à un autre signal $u(t)$ peut être calculee par une simple convolution :

$$
p(t)=p_{s}(t) * u(t)
$$

où $\mathrm{p}(\mathrm{t})$ dénote la pression acoustique rétrodiffusée au point d'observation. A titre d'exemple, le champ diffusé a été calculé pour deux signaux, toujours avec a $=0,5 \mathrm{~cm}, \mathrm{r}_{0}=20 \mathrm{~cm}$ et $\mathrm{c}=331 \mathrm{~m} / \mathrm{s}$.

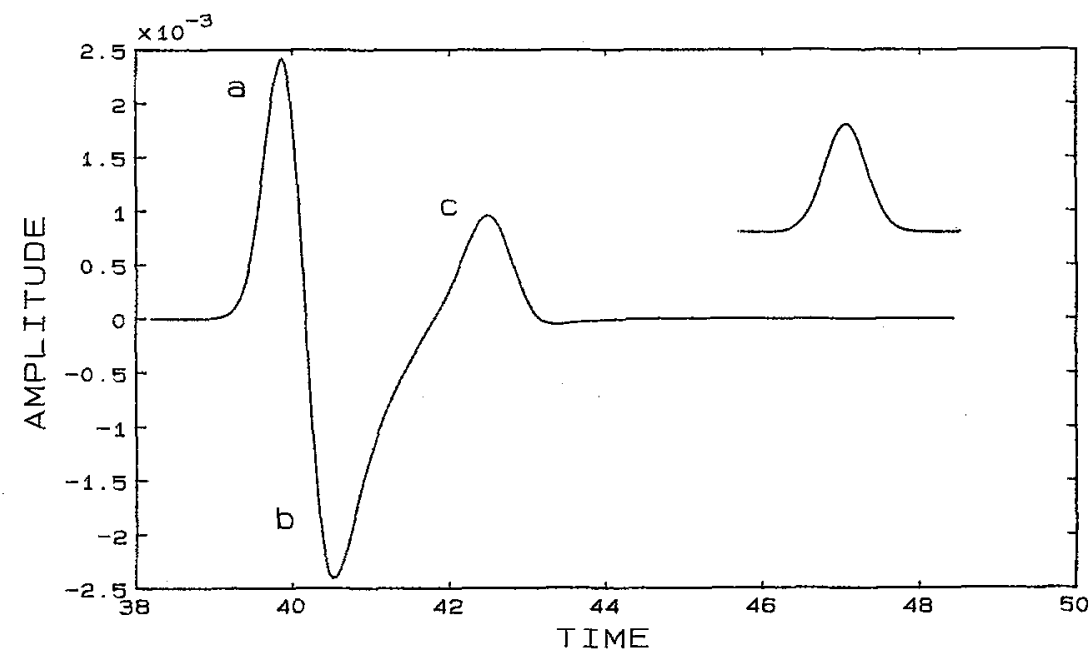

Figure 6: Réponse impulsionnelle à un signal incident gaussien. L'onde incidente correspondante est représentée en haut et à droite de la figure.

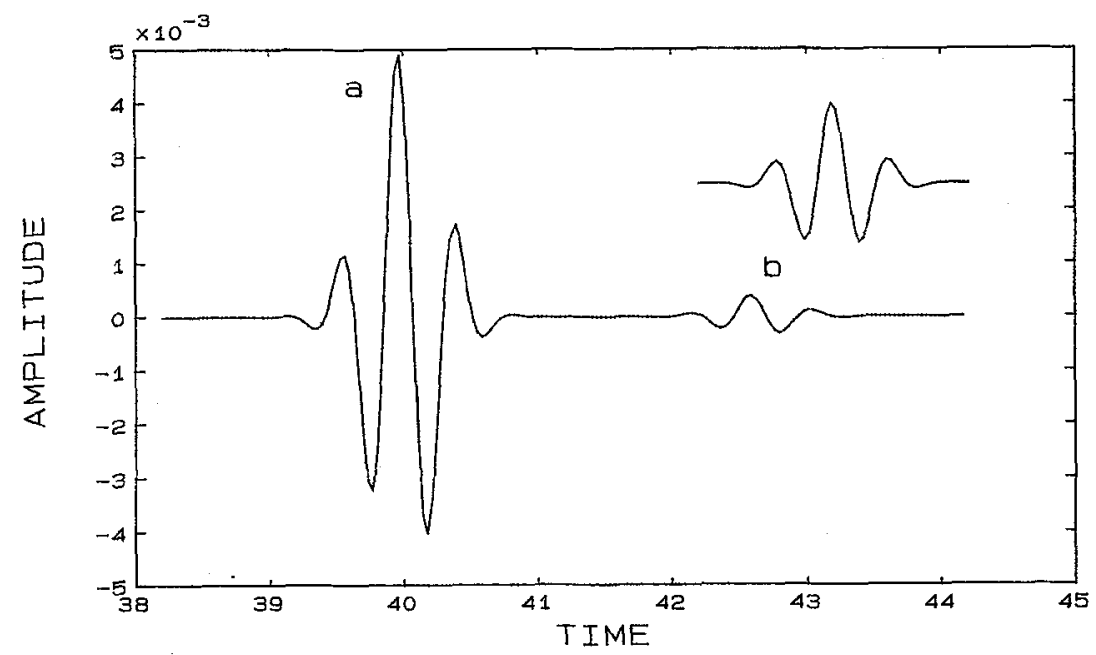

Figure 7: Réponse à un signal incident sinusoïdal dont l'amplitude est modulée par un gaussien. L'onde incidente correspondante est représentée en haut et à droite de la figure.

Le premier correspond à une émission gaussienne (pour simplifier, nous avons pris l'unité pour le coefficient de la fonction exponentielle) : 


$$
u(t)=\left\{\begin{array}{lc}
\exp [-\mathrm{q}(\mathrm{t}-\mathrm{e} \tau)]^{2} & 0 \leqslant t \leqslant 2 \mathrm{e} \tau \\
0 & \text { ailleurs }
\end{array}\right.
$$

et le deuxième est un signal sinusoïdal dont l'amplitude est modulée par $u(t)$ :

$$
s(t)= \begin{cases}\exp [-q(t-e \tau)]^{2} \sin (2 \pi f t) & 0 \leqslant t \leqslant 2 e \tau \\ 0 & \text { ailleurs }\end{cases}
$$

où $\tau=2 \mathrm{a} / \mathrm{c}, \mathrm{q}$, e sont des constantes et $\mathrm{f}$ est la fréquence de la porteuse de l'onde incidente. Dans les figures (6) et (7) sont représentés, pour $q=87384$ et $\mathrm{e}=1$, les signaux correspondants aux pressions rétrodiffusées, ainsi que les signaux émis. Dans notre calcul, la porteuse est à la fréquence de $75 \mathrm{kHz}$. Comme nous pouvons le voir, les contributions dues aux ondes rampantes (partie $\mathrm{c}$ dans la figure 6 et partie $\mathrm{b}$ dans la figure 7) sont aisées à identifier.

\section{Conclusion}

Dans ce travail nous avons présenté une méthode composite basée sur la combinaison de la méthode de l'optique physique et de la méthode modale pour calculer la réponse à une onde incidente en forme de Dirac, rétrodiffusée par une sphère rigide. A partir de la réponse impulsionnelle obtenue, la réponse à d'autres signaux peut être calculée immédiatement par convolution. En principe, la même méthode peut être étendue au calcul de la réponse d'autres objets rigides ou mous, de forme géométrique simple, pour laquelle l'équation de propagation acoustique est séparable dans le système de coordonnés approprié[1], aussi bien dans le cas de la rétrodiffusion que dans une situation bistatique.

En résumé les idées à la base de la méthode présentée sont :

1. Quand la fréquence est suffisamment élevée, la méthode modale et la méthode de l'optique physique tendent à donner le même résultat.

2. La réponse temporelle correspondant à la méthode d'optique physique doit être calculable [cf. Eq.(35)].

Au sujet du deuxième point, remarquons que pour un objet rigide ou mou, sous le postulat de l'optique physique, l'intégration de l'équation (3) dans le domaine fréquentiel peut être réalisée de $-\infty$ à $+\infty$. Ainsi la réponse temporelle dans le cadre de l'optique physique peut être obtenue et notre méthode est applicable dans cette situation.

\section{REMERCIEMENTS}

Ce travail a été réalisé dans le cadre d'une collaboration avec IFREMER.

\section{REFERENCES}

[1] J. Bowman, T. Senior and P.Uslenghi, Electromagnetic and Acoustic Scattering by Simple Shapes (North-Holland, Amsterdam, 1969).

[2] C.S. Clay and H. Medwin, Acoustical Oceanography (John Wiley \& Sons, New York, 1977), pp.502-506.

[3] J. Bowman, T. Senior and P.Uslenghi, Electromagnetic and Acousti Scattering by Simple Shapes (North-Holland, Amsterdam, 1969), p. 29

[4] M. Jessel, Acoustique Théorique : Propagation et Holophonie (Masson, Paris, 1973), p. 55.

[5] H. \& B.S. Jeffreys, Mathematical Physics (University Press, Cambridge, 1956), P. 659.

[6] P. M. Morse and K.U. Ingard, Theoretical Acoustics (Mc Graw-Hill, New York,1968), p. 339. 